TRANSACTIONS OF THE

AMERICAN MATHEMATICAL SOCIETY

Volume 350, Number 7, July 1998, Pages 2797-2811

S 0002-9947(98)02208-9

\title{
A HILBERT-NAGATA THEOREM IN NONCOMMUTATIVE INVARIANT THEORY
}

\author{
MÁTYÁS DOMOKOS AND VESSELIN DRENSKY
}

\begin{abstract}
Nagata gave a fundamental sufficient condition on group actions on finitely generated commutative algebras for finite generation of the subalgebra of invariants. In this paper we consider groups acting on noncommutative algebras over a field of characteristic zero. We characterize all the T-ideals of the free associative algebra such that the algebra of invariants in the corresponding relatively free algebra is finitely generated for any group action from the class of Nagata. In particular, in the case of unitary algebras this condition is equivalent to the nilpotency of the algebra in Lie sense. As a consequence we extend the Hilbert-Nagata theorem on finite generation of the algebra of invariants to any finitely generated associative algebra which is Lie nilpotent. We also prove that the Hilbert series of the algebra of invariants of a group acting on a relatively free algebra with a non-matrix polynomial identity is rational, if the action satisfies the condition of Nagata.
\end{abstract}

\section{INTRODUCTION}

Throughout this paper $K$ denotes a field of characteristic zero. Let $V$ be a finite dimensional vector space of dimension $m \geq 2$ over $K$. Denoting by $x_{1}, \ldots, x_{m}$ a linear basis of $V$, the tensor algebra $K\langle V\rangle$ of $V$ can be identified with the free unitary associative algebra $K\left\langle x_{1}, \ldots, x_{m}\right\rangle$.

The action of the general linear group $G L(V)$ extends naturally to the diagonal action of $G L(V)$ on $K\langle V\rangle$. Let $I$ be a $T$-ideal of $K\langle V\rangle$, i.e. $I$ is an ideal which is closed under all $K$-algebra endomorphisms of $K\langle V\rangle$. The factor algebra $K\langle V\rangle / I$ is the relatively free algebra of rank $m$ in the variety of unitary associative algebras satisfying all the polynomial identities from $I$. Since $I$ is a $G L(V)$-submodule of $K\langle V\rangle$, the algebra $K\langle V\rangle / I$ inherits the $G L(V)$-module structure of $K\langle V\rangle$.

Let $G$ be a subgroup of $G L(V)$. We shall study the algebra of invariants of $G$

$$
(K\langle V\rangle / I)^{G}=\{f \in K\langle V\rangle / I \mid g \cdot f=f \text { for all } g \in G\} .
$$

In the special case when $I$ is the commutator ideal of $K\langle V\rangle$, we get that $K\langle V\rangle / I$ is isomorphic to the symmetric algebra $K[V]$ of $V$ (or, in other words, $K[V]=$

Received by the editors June 5, 1996

1991 Mathematics Subject Classification. Primary 16W20; Secondary 16R10, 16P40, 16W50, 13A50, 15A72.

Key words and phrases. T-ideals, algebras with polynomial identity, noncommutative invariant theory, algebra of invariants, rational Hilbert series.

The first author was partially supported by Hungarian National Foundation for Scientific Research Grant no. F023436.

The second author was partially supported by Grant MM605/96 of the Bulgarian Foundation for Scientific Research. 
$K\left[x_{1}, \ldots, x_{m}\right]$ is the polynomial algebra in $m$ commuting variables over $\left.K\right)$. The investigation of $K[V]^{G}$ is the topic of classical invariant theory. On the other hand, the noncommutative invariant theory deals with $(K\langle V\rangle / I)^{G}$ for arbitrary T-ideals $I$. As in other recent branches of mathematics, one of the main problems here is to find reasonable limits for generalization of the classical results in the new situation. A survey on noncommutative invariant theory can be found in [8], [7].

The famous Hilbert-Noether theorem asserts that $K[V]^{G}$ is finitely generated for any finite group $G$. Kharchenko [11] extended this result to the noncommutative case and showed that $(K\langle V\rangle / I)^{G}$ is finitely generated for any finite group $G$ if and only if $K\langle V\rangle / I$ is weakly Noetherian, i.e. $K\langle V\rangle / I$ satisfies the ascending chain condition on two-sided ideals. These T-ideals have many other characterizations in the languages of polynomial identities, of noncommutative invariant theory and even of algorithmic problems in algebra, see for example the surveys [8], [7] an [12].

Nagata (see [4, Chapter 3]) found a fundamental condition assuring the finite generation of $K[V]^{G}$. This condition applies to a large class of group actions, including all rational representations of reductive linear algebraic groups.

The main purpose of our paper is the extension of the Hilbert-Nagata theorem to the noncommutative case. We describe all T-ideals $I$ such that the algebra of invariants $(K\langle V\rangle / I)^{G}$ is finitely generated for all groups $G$ from the class of Nagata. It turns out that all these relatively free algebras $K\langle V\rangle / I$ satisfy the identity of Lie nilpotency $\left[x_{1}, \ldots, x_{n}\right]=0$ for some $n$. As a consequence we obtain that if $R$ is any finitely generated associative algebra which is Lie nilpotent and $G$ is a group of automorphisms of $R$ satisfying the conditions of Nagata, then the algebra of fixed points $R^{G}$ is finitely generated. We note that since Lie nilpotent finitely generated algebras are Noetherian by [14], this latter result essentially follows from the following theorem of Vonessen [18]: If $G$ is a linearly reductive algebraic group acting rationally on a left Noetherian finitely generated PI-algebra $R$, then $R^{G}$ is finitely generated.

Afterwards, we investigate the question of rationality of the Hilbert series of the algebra of invariants in the noncommutative case. We show that $(K\langle V\rangle / I)^{G}$ has a rational Hilbert series for any group $G$ satisfying the condition of Nagata if $I$ contains a non-matrix identity. In particular this covers all the cases when $(K\langle V\rangle / I)^{G}$ is finitely generated by our theorem.

In the final section we consider T-ideals of the free non-unitary algebra $K_{+}\langle V\rangle$. Again, we give a complete description of the T-ideals of $K_{+}\langle V\rangle$ such that finite generation of the invariant algebra in the corresponding non-unitary relatively free algebra holds for any group action satisfying the condition of Nagata. These Tideals are characterized by the property that they are contained neither in the square of the commutator ideal nor in the T-ideal generated by the polynomial $x_{1}\left[x_{2}, x_{3}\right] x_{4}$. In particular, we obtain examples of non-Noetherian varieties, where the Hilbert-Nagata theorem holds.

\section{Preliminaries}

In this section we consider the following general setting. Let $R$ be a finitely generated (not necessarily commutative or unitary) $K$-algebra and let $G$ be a group acting by algebra automorphisms on $R$. In the sequel we refer to the following condition on the action of $G$ on $R$ (respectively on the representation of $G$ on some finite dimensional vector space $W$ ) as (i) (respectively (ii)): 
(i) The orbit under $G$ of any $f \in R$ is contained in a finite dimensional $K$ subspace of $R$.

(ii) If $W$ contains a $G$-invariant hyperplane $H_{1}$ and $G$ acts identically on the factor space $W / H_{1}$, then there exists a non-zero element $v \in W$ fixed by $G$ and a $G$-invariant hyperplane $H_{2}$ such that $W=K v \oplus H_{2}$.

We say that the action of $G$ on $R$ satisfies condition (ii) if (ii) holds for any finite dimensional subrepresentation of $G$ in $R$.

Denote by $R^{G}=\{f \in R \mid g \cdot f=f$ for every $g \in G\}$ the algebra of fixed points (or the algebra of invariants) of the action of $G$ on $R$.

Hilbert-Nagata Theorem. Let $R$ be a finitely generated commutative $K$-algebra, and let $G$ act on $R$ by algebra automorphisms. If the action of $G$ on $R$ satisfies (i) and finite dimensional representations of $G$ satisfy (ii), then $R^{G}$ is a finitely generated $K$-algebra.

Remark. Note that the most important corollary of the proof of this theorem is that $R^{G}$ is finitely generated if $G$ is a reductive linear algebraic group acting rationally on $R$, that is, the action is induced by a rational representation of the algebraic group $G$ on some finite dimensional subspace of $R$ generating the whole algebra.

Now we give a series of statements which are straightforward generalizations of statements used in Nagata's proof of the above theorem. Their commutative version can be found for example in [4, Chapter 3]. We assume that $R$ is a finitely generated (not necessarily commutative or unitary) $K$-algebra and $G$ acts on $R$ in such a way that the conditions (i) and (ii) hold.

Lemma 2.1. For any $f \in R$ there exists an $f^{*} \in R^{G} \cap \sum_{g \in G} K g \cdot f$ such that $f-f^{*} \in \sum_{g, h \in G} K(g \cdot f-h \cdot f)$.

Proof. The statement of the lemma concerns only the finite dimensional representation of $G$ generated by $f$ and does not involve any other algebraic properties of $R$. Therefeore the same proof works as for [4, Chapter 3, Lemma 1].

In the next two statements $M$ is a left $R$-module, and we assume that $G$ acts on $M$ by $K$-linear transformations such that

$$
g \cdot(r a)=(g \cdot r)(g \cdot a)
$$

for every $g \in G, r \in R$ and $a \in M$. We denote

$$
M^{G}=\{a \in M \mid g \cdot a=a \text { for every } g \in G\} .
$$

Lemma 2.2. The following equality holds in the $R$-submodule $\sum_{i=1}^{r}\left(R m_{i}+K m_{i}\right)$ generated by $m_{1}, \ldots, m_{r} \in M^{G}$ :

$$
M^{G} \cap \sum_{i=1}^{r}\left(R m_{i}+K m_{i}\right)=\sum_{i=1}^{r}\left(R^{G} m_{i}+K m_{i}\right) .
$$

Proof. The proof of [4, Chapter 3, Lemma 2] uses only the left $R$-module structure of $R$ and everything works under our hypotheses.

The above lemma has the following immediate consequence.

Corollary 2.3. If $M$ is a Noetherian R-module, then $M^{G}$ is finitely generated as an $R^{G}$-module. 
Lemma 2.4. Assume that $G$ acts on the finitely generated $K$-algebras $R_{1}$ and $R_{2}$ such that the action of $G$ on $R_{1}$ satisfies the conditions (i) and (ii). If $\phi: R_{1} \rightarrow$ $R_{2}$ is a surjective $G$-equivariant homomorphism, then $\left.\phi\right|_{R_{1}^{G}}: R_{1}^{G} \rightarrow R_{2}^{G}$ is also surjective.

Proof. Take any element of $R_{2}^{G}$. By the surjectivity of $\phi$ it is the image of some $f \in R_{1}$. By Lemma 2.1 we may write $f=f^{*}+f^{\prime}$, where $f^{*} \in R_{1}^{G}$ and $f^{\prime}=$ $\sum_{g, h \in G} \alpha_{g, h}(g \cdot f-h \cdot f)$, with $\alpha_{g, h} \in K$. Then

$$
\begin{aligned}
\phi\left(f-f^{*}\right) & =\phi\left(f^{\prime}\right)=\phi\left(\sum_{g, h \in G} \alpha_{g, h}(g \cdot f-h \cdot f)\right) \\
& =\sum_{g, h \in G} \alpha_{g, h}(g \cdot \phi(f)-h \cdot \phi(f))=0,
\end{aligned}
$$

since $\phi(f) \in R_{2}^{G}$. Hence $\phi(f)=\phi\left(f^{*}\right)$, where $f^{*} \in R_{1}^{G}$.

The proof of [4, Chapter 3, Lemma 3] does not use the commutativity of $R$, so the same argument gives the following assertion.

Lemma 2.5. Let $R=\sum_{i=0}^{\infty} R_{i}$ be a graded $K$-algebra with $R_{0}=K$. If the ideal $R_{+}=\sum_{i \geq 1} R_{i}$ is finitely generated as a two-sided ideal, then $R$ is finitely generated as a $K$-algebra.

Recall that an automorphism $\phi$ of a graded $K$-algebra $R=\sum_{i \geq 0} R_{i}$ is graded if $\phi\left(R_{i}\right)=R_{i}, i \geq 0$.

Proposition 2.6. Let $R=\sum_{i=0}^{\infty} R_{i}$ be a finitely generated graded $K$-algebra (with $\left.R_{0}=K\right)$, which is left Noetherian (i.e. $R$ is Noetherian as a left $R$-module). Assume, that $G$ acts on $R$ by graded automorphisms such that the conditions (i) and (ii) are satisfied. Then $R^{G}$ is a finitely generated $K$-algebra.

Proof. It is again a word by word repetition of the proof of the commutative case. Since the elements of $G$ are graded automorphisms, $R^{G}$ is also graded. The left $R$ submodule $R R_{+}^{G}+R_{+}^{G}$ of $R$ is finitely generated, since $R$ is left Noetherian. Hence by Lemma $2.2, R_{+}^{G}$ is a finitely generated $R^{G}$-module and, by Lemma $2.5, R^{G}$ is a finitely generated $K$-algebra.

Remark. Obviously the same conclusion holds if the $K$-algebra $R$ is right Noetherian.

Let $M=\sum_{d=0}^{\infty} M_{d}$ be a graded vector space such that $\operatorname{dim}_{K}\left(M_{d}\right)$ is finite for all $d$. We associate with $M$ a formal power series with integer coefficients

$$
H(M ; t)=\sum_{d=0}^{\infty} \operatorname{dim}_{K}\left(M_{d}\right) t^{d} \in \mathbb{Z}[[t]]
$$

which is called the Hilbert series of $M$. We say that $H(M ; t)$ is rational if there exist some polynomials $P(t), Q(t) \in \mathbb{Z}[t]$ such that $Q(t) H(M ; t)=P(t)$ in $\mathbb{Z}[[t]]$, and we write $H(M ; t)=\frac{P(t)}{Q(t)}$ in this case. We shall use the following well known theorem (see e.g. [3, Ch.11]).

Theorem 2.7 [Hilbert-Serre]. Let $R$ be a commutative graded algebra generated by a finite set $u_{1}, \ldots, u_{r}$ of homogeneous elements of degree $d_{1}, \ldots, d_{r}$, respectively, 
and let $M$ be a finitely generated graded $R$-module. Then the Hilbert series $H(M ; t)$ is rational and there exists a polynomial $F(t) \in \mathbb{Z}[t]$ such that

$$
H(M ; t)=\frac{F(t)}{\prod_{i=1}^{r}\left(1-t^{d_{i}}\right)} .
$$

\section{Noncommutative Hilbert-Nagata Theorem}

Denote by $K\left\langle x_{1}, x_{2}, \ldots\right\rangle$ the free associative algebra of countably infinite rank. For simplicity of the notation sometimes we shall also use other symbols, e.g. $x, y, z, y_{i}, z_{j}$, etc. instead of $x_{k}$ to denote different noncommutative variables.

We define long commutators recursively by

$$
[x, y]=x y-y x
$$

and

$$
\left[x_{i_{1}}, \ldots, x_{i_{n}}\right]=\left[\left[x_{i_{1}}, \ldots, x_{i_{n-1}}\right], x_{i_{n}}\right]
$$

for $n \geq 3$. The commutator ideal

$$
C=([u, v] \mid u, v \in K\langle V\rangle)
$$

is the two-sided ideal of $K\langle V\rangle$ generated by all the commutators.

It is known that the following conditions are equivalent for a T-ideal $I$ of $K\langle V\rangle$ :

1. The algebra $K\langle V\rangle / I$ is left Noetherian;

2. The algebra $K\langle V\rangle / I$ is right Noetherian;

3. The algebra $K\langle V\rangle / I$ satisfies some Engel identity $[y, x, \ldots, x]=0$;

4. The algebra $K\langle V\rangle / I$ satisfies the identity $\left[x_{1}, \ldots, x_{n}\right]=0$ for some $n$.

The equivalence $(1) \Leftrightarrow(3) \Leftrightarrow(2)$ was shown by Latyshev in [14]. In the same paper he proved that any finitely generated PI-algebra satisfying a non-matrix polynomial identity, satisfies also all the identities of some power of $C$. Applying this result to $K\langle V\rangle / I$ we obtain that $K\langle V\rangle / I$ is solvable as a Lie algebra, and, by a theorem of Higgins [9] $K\langle V\rangle / I$ is Lie nilpotent. (Actually Zelmanov [19] proved the stronger result that any Lie algebra (over a field of characteristic zero) satisfying the Engel identity is nilpotent.) The implication $(4) \Rightarrow(3)$ is trivial.

Theorem 3.1. The following conditions are equivalent for a T-ideal I of the free algebra $K\langle V\rangle$, where $\operatorname{dim}_{K}(V) \geq 2$.

1. The algebra of invariants $(K\langle V\rangle / I)^{G}$ is finitely generated for any group $G \leq$ $G L(V)$ whose action on $K\langle V\rangle / I$ satisfies the condition (ii);

2. The algebra $K\langle V\rangle / I$ satisfies the polynomial identity $\left[x_{1}, \ldots, x_{n}\right]=0$ for some $n$.

Proof. (1) $\Rightarrow(2)$ : It is well known that for any T-ideal $I$ of $K\langle V\rangle$ either $K\langle V\rangle / I$ satisfies $\left[x_{1}, \ldots, x_{n}\right]=0$ for some $n$ or $I$ is contained in $C^{2}$. (Indeed, if $I$ is not contained in $C^{2}$, then $I+C^{2}$ contains some non-zero linear combination of long commutators, hence $I$ contains an Engelian element $\left[x_{2}, x_{1}, \ldots, x_{1}\right]$.) In the latter case $K\langle V\rangle / C^{2}$ is a homomorphic image of $K\langle V\rangle / I$, so by Lemma 2.4 it suffices to show a group $G \leq G L(V)$ such that $\left(K\langle V\rangle / C^{2}\right)^{G}$ is not finitely generated, and the action of $G$ satisfies (ii). We claim that the algebra $\left(K\left\langle x_{1}, \ldots, x_{m}\right\rangle / C^{2}\right)^{G}$ is not finitely generated where $G$ is the torus

$$
G=\left\{\operatorname{diag}\left(\nu, \nu^{-1}, 1, \ldots, 1\right) \mid \nu \in K^{*}\right\} .
$$


In order to prove the claim we can use an example given by Kharchenko in [11]. Consider the factor algebra $K\langle x, y\rangle /(y x)$ of the free algebra $K\langle x, y\rangle$ modulo the two-sided ideal generated by $y x$. It satisfies the identity $\left[x_{1}, x_{2}\right]\left[x_{3}, x_{4}\right]=0$, so there exists a unique homomorphism

$$
\phi: K\left\langle x_{1}, \ldots, x_{m}\right\rangle / C^{2} \rightarrow K\langle x, y\rangle /(y x)
$$

such that

$$
\begin{aligned}
x_{1}+C^{2} & \rightarrow \bar{x}=x+(y x) \\
x_{2}+C^{2} & \rightarrow \bar{y}=y+(y x) \\
x_{3}+C^{2} & \rightarrow 0 \\
\vdots & \\
x_{m}+C^{2} & \rightarrow 0 .
\end{aligned}
$$

We define the action of $G$ on $K\langle x, y\rangle /(y x)$ in the obvious way. Then $\phi$ is $G$ equivariant and Lemma 2.4 gives that $\phi\left(\left(K\left\langle x_{1}, \ldots, x_{m}\right\rangle / C^{2}\right)^{G}\right)=(K\langle x, y\rangle /(y x))^{G}$. Hence it is sufficient to show that the latter is not finitely generated. $\left\{\bar{x}^{k} \bar{y}^{k} \mid k=\right.$ $0,1, \ldots\}$ is a $K$-linear base of $(K\langle x, y\rangle /(y x))^{G}$, so it is infinite dimensional. On the other hand, the product of any two non-constant basis elements is zero, showing that $(K\langle x, y\rangle /(y x))^{G}$ is not finitely generated.

$(2) \Rightarrow(1)$ : We may apply Proposition 2.6 with $R=K\langle V\rangle / I$.

Remark. There are examples with different groups acting on $K\langle V\rangle / C^{2}$ such that the conditions (i) and (ii) hold, however, the algebra of invariants is not finitely generated. For example, it can be shown that $\left(K\left\langle x_{1}, x_{2}\right\rangle / C^{2}\right)^{S L_{2}}$ is not finitely generated, with the canonical action of the special linear group $S L_{2}$ on the 2dimensional vector space $V$.

Corollary 3.2. Let $R$ be a finitely generated $K$-algebra satisfying some Engel identity $[y, x, \ldots, x]=0$. If the action of $G$ on $R$ satisfies the condition (i) and any finite dimensional representation of $G$ satisfies (ii), then the algebra of fixed points $R^{G}$ is finitely generated. (In particular, if $G$ is a reductive linear algebraic group acting rationally on $R$, then $R^{G}$ is finitely generated.)

Proof. Take any finite set of generators of $R$, it is contained in a finite dimensional $G$-invariant subspace $V$ of $R$ by (i), and any basis $b_{1}, \ldots, b_{m}$ of this space forms another generating set of the algebra. The action of $G$ on $V$ induces an action on $K\langle V\rangle=K\left\langle x_{1}, \ldots, x_{m}\right\rangle$, and the homomorphism

$$
K\left\langle x_{1}, \ldots, x_{m}\right\rangle \rightarrow R
$$

defined by

$$
x_{i} \rightarrow b_{i} \quad(i=1, \ldots, m)
$$

is $G$-equivariant and factors through the algebra $K\left\langle x_{1}, \ldots, x_{m}\right\rangle / I$, where $I$ is the intersection of $K\left\langle x_{1}, \ldots, x_{m}\right\rangle$ with the T-ideal generated by $[y, x, \ldots, x]$. Therefore $R^{G}$ is a homomorphic image of $\left(K\left\langle x_{1}, \ldots, x_{m}\right\rangle / I\right)^{G}$ by Lemma 2.4, and the latter is finitely generated by Theorem 3.1 . 
Remark. It is clear that the same conclusion holds under the weaker condition that the action of $G$ on $K\langle V\rangle / I$ satisfies (ii), where $V$ and $I$ are the same as in the proof of Corollary 3.2.

One may paraphrase our results in the following way. The Hilbert-Nagata theorem holds in a variety of associative unitary $K$-algebras if and only if the variety is Noetherian, that is, any finitely generated algebra in the variety is Noetherian. For comparison, recall that Kharchenko showed in [11] that Noether's theorem holds in a variety if and only if it is weakly Noetherian (and he noted also that the HilbertNagata theorem may fail in some weakly Noetherian varieties). This difference demonstrates that though the Hilbert Basis Theorem is a crucial ingredient of the proofs of these theorems in the commutative case, its role is a little bit different in the finite and the reductive group case.

\section{Rationality of the Hilbert series}

Let $f$ be an element of the free algebra $K\left\langle x_{1}, x_{2}, \ldots\right\rangle$. We say that $f=0$ is a non-matrix polynomial identity if it is not an identity for the $2 \times 2$ matrix algebra over $K$. The following conditions are equivalent for a T-ideal $I$ of $K\langle V\rangle$ (where $\operatorname{dim}_{K}(V) \geq 2$ ).

1. The algebra $K\langle V\rangle / I$ satisfies some non-matrix identity;

2. The ideal $I$ contains $\left[x_{1}, x_{2}\right]^{n}$ for sufficiently large $n$;

3. The algebra $K\langle V\rangle / I$ satisfies the identity $\left[x_{1}, x_{2}\right] \ldots\left[x_{2 k-1}, x_{2 k}\right]=0$ for sufficiently large $k$.

$(1) \Rightarrow(2)$ was proved by Amitsur in [2], $(2) \Rightarrow(3)$ is a result of Latyshev [14], and $(3) \Rightarrow(1)$ is trivial.

Theorem 4.1. Let $I$ be a T-ideal of $K\langle V\rangle$, and let $G$ be a subgroup of $G L(V)$ such that the action of $G$ on $K\langle V\rangle$ satisfies (ii). (In particular, $G$ may be a reductive linear algebraic subgroup of $G L(V)$.) If $K\langle V\rangle / I$ satisfies some non-matrix identity, then $(K\langle V\rangle / I)^{G}$ has a rational Hilbert series and

$$
H\left((K\langle V\rangle / I)^{G} ; t\right)=\frac{F(t)}{\prod_{i=1}^{r}\left(1-t^{d_{i}}\right)},
$$

where $F(t)$ is a polynomial with integer coefficients, $r \in \mathbb{N}$ and $d_{1}, \ldots, d_{r} \in \mathbb{N}$.

Proof. We use ideas of [5] and [13]. The ideal $I$ contains some power $C^{k+1}$ of the commutator ideal $C$ of $K\langle V\rangle$. For any exact sequence of graded vector spaces

$$
0 \rightarrow M_{1} \rightarrow M \rightarrow M_{2} \rightarrow 0
$$

the equality $H(M ; t)=H\left(M_{1} ; t\right)+H\left(M_{2} ; t\right)$ holds. Hence we have

$$
H\left((K\langle V\rangle / I)^{G} ; t\right)=\sum_{d=0}^{k} H\left(\left(C^{d} /\left(I \cap C^{d}+C^{d+1}\right)\right)^{G} ; t\right)
$$

and it suffices to establish the theorem for the case $C^{d+1} \subseteq I \subseteq C^{d}(d=0,1, \ldots)$. The result is known for $d=0$, because in this case $K\langle V\rangle / I \cong K[V]$. We assume that $d \geq 1$ and denote the images of $x_{1}, \ldots, x_{m}$ in $K\left\langle x_{1}, \ldots, x_{m}\right\rangle / C^{d+1}$ by the same symbols. It is also known that $C^{d} / C^{d+1}$ is spanned over $K$ by the elements

$$
w=w\left(x_{1}, \ldots, x_{m}\right)=x_{1}^{a_{1}} \ldots x_{m}^{a_{m}} \prod_{s=1}^{d}\left(\left[x_{i_{s}}, x_{j_{s}}\right] \prod_{k=1}^{m}\left(\operatorname{ad} x_{k}\right)^{b_{s k}}\right),
$$


where $a_{1}, \ldots, a_{m}, b_{s k}(1 \leq s \leq d, 1 \leq k \leq m)$ are non-negative integers, $i_{s}, j_{s} \in$ $\{1, \ldots, m\}$ and $x(\operatorname{ad} y)^{b}=[x, y, \ldots, y]$ (here the number of the $y$ 's is $b$ ). We define an action of the commutative polynomial algebra

$$
R=K\left[t_{1}, \ldots, t_{m}, u_{s k} \mid 1 \leq s \leq d, 1 \leq k \leq m\right]
$$

on $C^{d} / C^{d+1}$ by

$$
\prod_{i=1}^{m} t_{i}^{c_{i}} \prod_{s=1}^{d} \prod_{k=1}^{m} u_{s k}^{e_{s k}} \cdot w=x_{1}^{a_{1}+c_{1}} \ldots x_{m}^{a_{m}+c_{m}} \prod_{s=1}^{d}\left(\left[x_{i_{s}}, x_{j_{s}}\right] \prod_{k=1}^{m}\left(a d x_{k}\right)^{b_{s k}+e_{s k}}\right) .
$$

The group $G L(V)=G L_{m}$ acts on $R$ by

$$
g \cdot t_{j}=\sum_{i=1}^{m} g_{i j} t_{i}, g \cdot u_{s j}=\sum_{i=1}^{m} g_{i j} u_{s i}, g=\left(g_{i j}\right) \in G L_{m} .
$$

It is obvious that the action of $G L_{m}$ on the $R$-module $C^{d} / C^{d+1}$ satisfies $g \cdot(f h)=$ $(g \cdot f)(g \cdot h)$ for any $g \in G L_{m}, f \in R$ and $h \in C^{d} / C^{d+1}$. Let $R_{0}$ be the subalgebra of $R$ generated by $t_{1}, \ldots, t_{m}, e_{i}\left(u_{1 j}, \ldots, u_{d j}\right) \quad(1 \leq i \leq d, 1 \leq j \leq m)$, where $e_{i}$ is the $i$ th elementary symmetric function in $d$ variables. Denote by $R_{1}$ the $G L_{m}$-submodule of $R$ generated by $R_{0}$, so $R_{1}$ is the subalgebra of $R$ generated by $t_{1}, \ldots, t_{m}, g \cdot e_{i}\left(u_{1 j}, \ldots, u_{d j}\right) \quad\left(1 \leq i \leq d, 1 \leq j \leq m, g \in G L_{m}\right)$. For any $q \in K$, $g \in G L_{m}$ and $i=1, \ldots, m$ we have the equality

$$
w\left(x_{1}+q\left[x_{1}, g \cdot x_{i}\right], \ldots, x_{m}+q\left[x_{m}, g \cdot x_{i}\right]\right)=\prod_{s=1}^{d}\left(1+q g \cdot u_{s i}\right) \cdot w .
$$

Since $I$ is a T-ideal, by standard Vandermonde arguments we obtain that $I$ is an $R_{1}$-submodule of $C^{d}$.

Since $C^{d} / C^{d+1}$ is a finitely generated $R$-module and $R$ is a finitely generated $R_{0^{-}}$ module, we obtain that $C^{d} / C^{d+1}$ is also finitely generated as an $R_{1}$-module. Since $I / C^{d+1}$ is an $R_{1}$-submodule of $C^{d} / C^{d+1}$ this implies that $C^{d} / I$ is a Noetherian $R_{1}$-module. By Corollary 2.3 the $R_{1}^{G}$-module $\left(C^{d} / I\right)^{G}$ is finitely generated. Our conditions imply that the action of $G$ on $R_{1}$ also satisfies (ii), because any irreducible polynomial representation of $G L(V)$ is a direct summand of $K\langle V\rangle$. Hence $R_{1}^{G}$ is finitely generated by the commutative Hilbert-Nagata theorem, and Theorem 2.7 implies that $H\left(\left(C^{d} / I\right)^{G} ; t\right)$ has the desired form.

Remarks. The proof of the above theorem remains valid under the condition that the action of $G$ on $K[W]$ satisfies (ii) whenever the representation of $G$ on $W$ is equivalent to a direct sum of some copies of $V$.

The free algebra $K\langle V\rangle$ has a rational Hilbert series. It is shown in [1] that if $S L_{2} \rightarrow G L(V)$ is any irreducible rational representation, then the Hilbert series of $K\langle V\rangle^{S L_{2}}$ is algebraic, but usually it is not rational. Hence Theorem 4.1 is not an immediate consequence of the fact that $K\langle V\rangle / I$ has a rational Hilbert series whenever $I$ contains some non-matrix identity (see [5]).

The algebra $(K\langle V\rangle / I)^{G}$ has a rational Hilbert series when the conditions of Theorem 3.1 are satisfied, and hence the algebra of invariants is finitely generated. (Actually, in this case we can repeat verbatim the usual proof of Theorem 2.7, using that $K\langle V\rangle / I$ is left Noetherian.) Comparing the two theorems we assert that there are examples where the algebra of invariants is not finitely generated, however, it has a rational Hilbert series. 


\section{NON-UNITARY VARIETIES}

Denote by $K_{+}\langle V\rangle$ the subalgebra of $K\langle V\rangle$ consisting of polynomials with zero constant term. We define T-ideals of $K_{+}\langle V\rangle$ similarly to the case of $K\langle V\rangle$. An ideal $I$ of $K_{+}\langle V\rangle$ is called a T-ideal if it is closed under $K$-algebra endomorphisms of $K_{+}\langle V\rangle$ and $K_{+}\langle V\rangle / I$ is the relatively free algebra of rank $m=\operatorname{dim}_{K}(V)$ in the variety of non-unitary associative $K$-algebras satisfying the identities $f=0$ (with $f \in I)$.

Obviously, if $I$ is a proper T-ideal of $K\langle V\rangle$, then it is a T-ideal of $K_{+}\langle V\rangle$ too. It is also clear that $(K\langle V\rangle / I)^{G}=K+\left(K_{+}\langle V\rangle / I\right)^{G}$ for any $G \leq G L(V)$ and any $G$-invariant ideal $I$. So the difference between the unitary and the non-unitary case is that we have more T-ideals in the non-unitary case. The aim of this section is to decribe all the T-ideals of $K_{+}\langle V\rangle$ such that $\left(K_{+}\langle V\rangle / I\right)^{G}$ is finitely generated for all groups $G$ whose action satisfies (ii).

We recall that a relatively free algebra $K_{+}\langle V\rangle / I$ is weakly Noetherian, if it satisfies the ascending chain condition for two-sided ideals. As we mentioned in the introduction, Kharchenko in [11] extended the commutative Hilbert-Noether theorem on finite generation of the algebra of invariants of finite groups to weakly Noetherian varieties. It was shown by L'vov in [15] that $K_{+}\langle V\rangle / I$ (where $\operatorname{dim}_{K}(V) \geq 2$ ) is weakly Noetherian if and only if $I$ contains an element of the form

$$
y x^{n} y-\sum_{i+j>0} a_{i j} x^{i} y x^{n-i-j} y x^{j}, \quad a_{i j} \in K .
$$

The next characterization of weakly Noetherian relatively free algebras is due to Mal'tsev [16]. We give an alternative proof which gives additional information on the weakly Noetherian varieties and is of independent interest. Throughout the section $U$ denotes the T-ideal of $K_{+}\left\langle x_{1}, x_{2}, \ldots\right\rangle$ generated by $x_{1}\left[x_{2}, x_{3}\right] x_{4}$.

Proposition 5.1. Let $I$ be a T-ideal of $K_{+}\langle V\rangle$ where $\operatorname{dim}_{K}(V) \geq 2$. Then the algebra $K_{+}\langle V\rangle / I$ is weakly Noetherian if and only if $I$ is not contained in $U$.

Consider $K_{+}\langle V\rangle=K_{+}\left\langle x_{1}, \ldots, x_{m}\right\rangle$ as a $G L_{m}$-module. The isomorphism types of the irreducible summands of the submodule of homogeneous polynomials of degree $n$ are parametrized by partitions of $n$ into not more than $m$ parts. Let $\lambda=\left(\lambda_{1}, \ldots, \lambda_{r}\right), r \leq m$, be a partition of $n$ (we use the notation $\lambda \vdash n$ ), i.e. $\lambda_{1} \geq \ldots \geq \lambda_{r} \geq 0$ are integers with $\lambda_{1}+\ldots+\lambda_{r}=n$. For any partition $\lambda=\left(\lambda_{1}, \ldots, \lambda_{r}\right) \vdash n, r \leq m$, we denote by $M(\lambda)$ the isomorphism type of the irreducible $G L(m)$-module corresponding to $\lambda$. We have

$$
K_{+}\left\langle x_{1}, \ldots, x_{m}\right\rangle /\left(U \cap K_{+}\left\langle x_{1}, \ldots, x_{m}\right\rangle\right) \cong \sum_{n=1}^{\infty} \sum_{\lambda \vdash n} n_{\lambda} M(\lambda)
$$

as $G L_{m}$-modules, where $n_{\lambda}$ denotes the multiplicity of the irreducible module $M(\lambda)$. We denote by $A$ the following subalgebra of the $3 \times 3$ matrix algebra:

$$
A=\left\{\left(\begin{array}{ccc}
0 & a & b \\
0 & c & d \\
0 & 0 & 0
\end{array}\right) \mid a, b, c, d \in K\right\} .
$$

Lemma 5.2. The T-ideal of the polynomial identities of $A$ coincides with $U$. The multiplicities $n_{\lambda}$ of $M(\lambda)$ in the relatively free algebra associated with $U$ are the 
following:

$$
n_{\lambda}= \begin{cases}1, & \text { if } \quad \lambda=(n) \quad(n \geq 1) \\ 2, & \text { if } \lambda=(n-1,1) \quad(n \geq 3) \\ 1, & \text { if } \lambda=(n-2,2) \quad(n \geq 4) \\ 1, & \text { if } \lambda=(n-2,1,1) \quad(n \geq 3) \\ 1, & \text { if } \lambda=(1,1) \\ 0, & \text { in all other cases. }\end{cases}
$$

Proof. Since $U$ contains no element of degree smaller than 4, the multiplicity of $M(\lambda)$ is the same as in the free algebra when $\lambda \vdash 1, \lambda \vdash 2$ or $\lambda \vdash 3$. We denote by $x_{i}$ both the generators of $K\langle V\rangle$ and their images in $K\langle V\rangle /(U \cap K\langle V\rangle)$. Consider any monomial

$$
x_{i_{0}} x_{i_{1}} \ldots x_{i_{r}} x_{i_{r+1}} \in K\langle V\rangle /(U \cap K\langle V\rangle),
$$

where $r \geq 2$. Using the identity $x[y, z] v=0$ we may exchange any two variables closed between $x_{i_{0}}$ and $x_{i_{r+1}}$ and, hence we may assume that $i_{1} \leq \ldots \leq i_{r}$. Therefore as a $G L_{m}$-module, $K\langle V\rangle /(U \cap K\langle V\rangle)$ is a factor module of $V \otimes K[V] \otimes V$. Applying the branching rule we compute the multiplicities of the irreducible components of $V \otimes K[V] \otimes V$, and we conclude that the values given in the statement of the lemma are upper bounds for $n_{\lambda}$.

One can easily check that the algebra $A$ satisfies the identity $x_{1}\left[x_{2}, x_{3}\right] x_{4}=0$. Hence its T-ideal contains $U$. Let $S_{k}$ be the symmetric group of degree $k$. For a fixed $n \geq 4$ we consider the following elements in $K_{+}\left\langle x_{1}, \ldots, x_{m}\right\rangle$ :

$$
\begin{aligned}
f_{1} & =x_{1}^{n}, \\
f_{2} & =x_{1}^{n-2}\left[x_{2}, x_{1}\right]=-\sum_{\sigma \in S_{2}}(\operatorname{sign} \sigma) x_{1}^{n-2} x_{\sigma(1)} x_{\sigma(2)}, \\
f_{3} & =\left[x_{2}, x_{1}\right] x_{1}^{n-2}=-\sum_{\sigma \in S_{2}}(\operatorname{sign} \sigma) x_{\sigma(1)} x_{\sigma(2)} x_{1}^{n-2}, \\
f_{4} & =\left[x_{2}, x_{1}\right] x_{1}^{n-4}\left[x_{2}, x_{1}\right]=\sum_{\sigma \in S_{2}} \sum_{\tau \in S_{2}}(\operatorname{sign} \sigma)(\operatorname{sign} \tau) x_{\sigma(1)} x_{\sigma(2)} x_{1}^{n-4} x_{\tau(1)} x_{\tau(2)}, \\
f_{5} & =\left[x_{2}, x_{1}\right] x_{1}^{n-4}\left[x_{3}, x_{1}\right]-\left[x_{3}, x_{1}\right] x_{1}^{n-4}\left[x_{2}, x_{1}\right] \\
& =\sum_{\sigma \in S_{3}}(\operatorname{sign} \sigma)\left[x_{\sigma(1)}, x_{1}\right] x_{1}^{n-4} x_{\sigma(2)} x_{\sigma(3)} .
\end{aligned}
$$

The polynomials $f_{1}, f_{2}, f_{3}, f_{4}, f_{5}$ are highest weight vectors generating irreducible $G L_{m}$-submodules of $K\langle V\rangle$ isomorphic to $M(n), M(n-1,1), M(n-1,1), M(n-2,2)$ and $M(n-2,1,1)$, respectively. (For details see e.g. [6].) The substitution

$$
x_{1} \rightarrow\left(\begin{array}{ccc}
0 & 0 & 0 \\
0 & \rho & 0 \\
0 & 0 & 0
\end{array}\right), x_{2} \rightarrow\left(\begin{array}{ccc}
0 & \eta & 0 \\
0 & 0 & \nu \\
0 & 0 & 0
\end{array}\right), x_{3} \rightarrow\left(\begin{array}{ccc}
0 & \eta^{\prime} & 0 \\
0 & 0 & \nu^{\prime} \\
0 & 0 & 0
\end{array}\right),
$$

$\rho, \eta, \nu, \eta^{\prime}, \nu^{\prime} \in K$, shows that $f_{1}, f_{4}, f_{5}$, and any linear combination of $f_{2}$ and $f_{3}$ are not contained in the T-ideal of identities of $A$. This proves both that the T-ideal of identities of $A$ equals $U$ and that the multiplicities $n_{\lambda}$ are as we claimed.

Proof of Proposition 5.1. First we show that if the T-ideal $I$ contains properly $U \cap$ $K\langle V\rangle$, then $K_{+}\langle V\rangle / I$ is weakly Noetherian. So assume that $U \cap K\langle V\rangle \subsetneq I$. Then 
$I$ contains $f_{1}, f_{4}, f_{5}$ or some linear combination of $f_{2}$ and $f_{3}$ (for suitable $n$ ), where $f_{i}$ are the polynomials introduced in the proof of Lemma 5.2. If $I$ contains $f_{1}$, then $K_{+}\langle V\rangle / I$ is nilpotent, and hence finite dimensional by the Dubnov-IvanovNagata-Higman theorem (see for example [10]). Any non-zero linear combination of $f_{2}$ and $f_{3}$ is of the form $(*)$. (Actually, we can say more. If $I$ contains some linear combination of $f_{2}$ and $f_{3}$, then it is left or right Noetherian by [14].) The polynomial $f_{4}$ again has the form $(*)$. If $I$ contains $f_{5}$, then it also contains

$$
f_{5}\left(x_{1}, x_{2}, x_{2} x_{1}\right)=\left[x_{2}, x_{1}\right] x_{1}^{n-4}\left[x_{2}, x_{1}\right] x_{1}-\left[x_{2}, x_{1}\right] x_{1} x_{1}^{n-4}\left[x_{2}, x_{1}\right] .
$$

Since $I$ contains $U \cap K\left\langle x_{1}, x_{2}, x_{3}\right\rangle$, we obtain that $I$ contains $\left[x_{2}, x_{1}\right] x_{1}^{n-3}\left[x_{2}, x_{1}\right]$, i.e. $I$ contains an identity of the form $(*)$.

Now assume that $I$ is any T-ideal of $K_{+}\langle V\rangle$ not contained in $U$. Then $I+$ $(U \cap K\langle V\rangle)$ contains properly $U \cap K\langle V\rangle$, hence $K_{+}\langle V\rangle /(I+(U \cap K\langle V\rangle))$ is weakly Noetherian, implying that $I+(U \cap K\langle V\rangle)$ contains an element of the form $(*)$. Any element in $U$ which depends on two variables only is a sum of polynomials

$$
f\left(x_{1}, x_{2}\right)\left[g\left(x_{1}, x_{2}\right), h\left(x_{1}, x_{2}\right)\right] l\left(x_{1}, x_{2}\right)
$$

$\left(f, g, h, l \in K_{+}\left\langle x_{1}, x_{2}\right\rangle\right)$, and it is easy to see that such an element does not contain the monomial $x_{2} x_{1}^{n} x_{2}$. Therefore $I$ itself contains an element of the form $(*)$, and hence $K_{+}\langle V\rangle / I$ is weakly Noetherian.

The above arguments show also that if $I$ is contained in $U$, then it does not contain an identity of the form $(*)$, implying that $K_{+}\langle V\rangle / I$ is not weakly Noetherian.

Proposition 5.3. Let $C$ be the commutator ideal of $K\left\langle x_{1}, x_{2}, \ldots\right\rangle$, and let $I$ be any T-ideal of $K_{+}\left\langle x_{1}, x_{2}, \ldots\right\rangle$. Then $I$ is not contained in $C^{2}$ if and only if it contains a multilinear identity

$$
x_{1} \ldots x_{N} y z_{1} \ldots z_{M}-\sum_{i=1}^{r} a_{i} v_{i} y w_{i}
$$

where $N, M, r$ are positive integers, $a_{i} \in K$, and $v_{i}, w_{i}$ are monomials such that the degree of $w_{i}$ is strictly smaller than $M(i=1, \ldots, r)$.

Proof. Any multilinear element of $C^{2}$ is a linear combination of polynomials

$$
x_{i_{1}} \ldots x_{i_{r}}\left[x_{j_{1}}, \ldots, x_{j_{s}}\right]\left[x_{k_{1}}, \ldots, x_{k_{t}}\right] .
$$

It is easy to see that for any variable $y$ of the above polynomial the number of monomials in which $y$ occurs in the $i$ th position is even for any $i$. Hence $C^{2}$ does not contain a polynomial of the form $(* *)$.

Now assume that $I$ is not contained in $C^{2}$. It is proved in [17] that $I$ contains $x^{k}\left(y(\operatorname{ad} x)^{l}\right) x^{p}$ for some positive integers $k, l, p$. (In order to see this, in [17] one should trace back the proof of Theorem 3.14 till Lemma 3.3, concluding that any polynomial identity not contained in $C^{2}$ has a consequence

$$
\sum_{i=0}^{n}(-1)^{i}\left(\begin{array}{c}
n \\
i
\end{array}\right) x^{n+i}[y, x] x^{2 n-i}=x^{n}\left(y(\operatorname{ad} x)^{n+1}\right) x^{n}
$$


modulo $C^{2}$. Since $C^{2}$ does not contain polynomials $f(x, y)$ which are linear in $y$, one obtains the statement.) In the equality

$$
x^{k}\left(y(\operatorname{ad} x)^{l}\right) x^{p}=x^{k} y x^{l+p}+\sum_{i=1}^{l} b_{i} x^{k+i} y x^{l+p-i}, \quad b_{i} \in K,
$$

we make the substitution $x \rightarrow x+z^{2}$, and take the multihomogeneous component of degree $k$ in $x$ of the resulting polynomial. We obtain that $I$ contains some polynomial

$$
x^{k} y z^{2(l+p)}+\sum_{i=1}^{s} b_{i}^{\prime} v_{i}^{\prime} y w_{i}^{\prime}
$$

where $\operatorname{deg}\left(w_{i}^{\prime}\right)<2(l+p),(i=1, \ldots, s)$. The linearization of the above polynomial in $z$ equals

$$
x^{k} y P\left(z_{1}, \ldots, z_{2(l+p)}\right)+\sum_{i=1}^{t} b_{i}^{\prime \prime} v_{i}^{\prime \prime} y w_{i}^{\prime \prime},
$$

where $P\left(x_{1}, \ldots, x_{n}\right)=\sum_{\sigma \in S_{n}} z_{\sigma(1)} \ldots z_{\sigma(n)}$. It is proved in [10] that there exists an integer $S$ such that $z_{1} \ldots z_{S}$ is a linear combination of some polynomials $P\left(u_{1}, \ldots, u_{2(l+p)}\right)$, where $u_{1}, \ldots, u_{2(l+p)}$ are monomials such that $u_{1} \ldots u_{2(l+p)}$ is multilinear in $z_{1}, \ldots, z_{S}$. Let us make the same substitutions in the above polynomial and take the same linear combination of the resulting polynomials, in order to obtain

$$
x^{k} y z_{1} \ldots z_{S}+\sum_{i=1}^{q} b_{i}^{\prime \prime \prime} v_{i}^{\prime \prime \prime} y w_{i}^{\prime \prime \prime},
$$

where $\operatorname{deg}\left(w_{i}^{\prime \prime \prime}\right)<S$. Now doing a similar process with the $x$ 's we can obtain a multihomogeneous element in $I$ of the form

$$
x_{1} \ldots x_{N} y z_{1} \ldots z_{S}+\sum_{i=1}^{n} a_{i} v_{i} y w_{i},
$$

where no $w_{i}$ contains all the variables $z_{1}, \ldots, z_{S}$. Let us substitute

$$
z_{i} \rightarrow z_{(i-1) j+1} \ldots z_{i j}, \quad(i=1, \ldots, S) .
$$

Choosing $j$ sufficiently large we obtain an identity of the form $(* *)$.

Lemma 5.4. Let the T-ideal I of $K_{+}\left\langle x_{1}, x_{2}, \ldots\right\rangle$ contain an element of the form $(*)$. Then for any positive integers $d$ and $m$ there exists a positive integer $N=$ $N(d, m)$ such that for any $i_{1}, \ldots, i_{N} \in\{1, \ldots, m\}$ and $0 \leq s_{1} \leq \ldots \leq s_{d-1} \leq N$ the T-ideal I contains a multihomogeneous element of the form

$$
\begin{aligned}
(* * *) \quad x_{1} y_{i_{1}} \ldots y_{i_{s_{1}}} & x_{2} y_{i_{s_{1}+1}} \ldots y_{i_{s_{2}}} x_{3} \ldots y_{i_{N}} x_{d} \\
& -\sum_{j=1}^{r} f_{j}\left(y_{1}, \ldots, y_{m}\right) g_{j}\left(x_{1}, \ldots, x_{d}, y_{1}, \ldots, y_{m}\right) h_{j}\left(y_{1}, \ldots, y_{m}\right),
\end{aligned}
$$

where $r \in \mathbb{N}$, the polynomials $f_{j}$ and $h_{j}$ do not depend on $x_{1}, \ldots, x_{d}$ and the degree of $g_{j}$ is strictly smaller than $N+d$ for $j=1, \ldots, r$. 
Proof. Let $\bar{K}$ be the algebraic closure of $K$. It is well known that the $\bar{K}$-vector subspace $\bar{I}=\bar{K} \otimes_{K} I$ of $\bar{K} \otimes_{K} K\left\langle x_{1}, x_{2}, \ldots\right\rangle \cong \bar{K}\left\langle x_{1}, x_{2}, \ldots\right\rangle$ is a T-ideal of $\bar{K}\left\langle x_{1}, x_{2}, \ldots\right\rangle$. Hence if we establish the statement for $\bar{K}$, then we immediately obtain it for $K$. Therefore, without loss of generality we may assume that the base field $K$ is algebraically closed. Let $\rho \in K$ be a primitive $d$ th root of unity and let $G$ be the subgroup of the automorphism group of $K_{+}\left\langle x_{1}, \ldots, x_{d}, y_{1}, \ldots, y_{m}\right\rangle$ generated by the automorphism

$$
\begin{aligned}
& x_{1} \rightarrow \rho x_{1}, \ldots, x_{d} \rightarrow \rho x_{d}, \\
& y_{1} \rightarrow y_{1}, \ldots, y_{m} \rightarrow y_{m} .
\end{aligned}
$$

By [15] and [11] the algebra $K_{+}\left\langle x_{1}, \ldots, x_{d}, y_{1}, \ldots, y_{m}\right\rangle / I$ is weakly Noetherian and its subalgebra of $G$-invariants is finitely generated. On the other hand, the monomials

$$
x_{1} y_{i_{1}} \ldots y_{i_{s_{1}}} x_{2} y_{i_{s_{1}+1}} \ldots y_{i_{s_{2}}} x_{3} \ldots y_{i_{N}} x_{d}
$$

are invariants of $G$ and, if $N$ is sufficiently large, they can be expressed with invariants of strictly lower degree. Clearly, a polynomial is an invariant of $G$ if and only if the total degree in the $x$ 's of each of its monomials is divisible by $d$. This means that $I$ contains polynomials of type $(* * *)$.

Now we are able to establish the main result of this section. We denote by $U$ and $C$ the same T-ideals as earlier in the section.

Theorem 5.5. For a T-ideal I of the non-unitary free algebra $K_{+}\langle V\rangle, \operatorname{dim}_{K}(V) \geq$ 2 , the following two conditions are equivalent.

1. The algebra of invariants $\left(K_{+}\langle V\rangle / I\right)^{G}$ is finitely generated for all subgroups $G$ of $G L(V)$ whose action on $K_{+}\langle V\rangle / I$ satisfies (ii);

2. The T-ideal $I$ is contained neither in $U$ nor in $C^{2}$.

Proof. (1) $\Rightarrow(2)$ : If $I$ is contained in $U$, then $K\langle V\rangle / I$ is not weakly Noetherian by Proposition 5.1 and hence $\left(K_{+}\langle V\rangle / I\right)^{G}$ is not finitely generated for some finite group $G$. We showed already in the proof of Theorem 3.1 that (1) implies that $I$ is not contained in $C^{2}$.

$(2) \Rightarrow(1)$ : If $I$ is not contained in $C$, then $K_{+}\langle V\rangle / I$ is finite dimensional by the Dubnov-Ivanov-Nagata-Higman theorem, and there is nothing to prove. So we may assume that $I$ is contained in $C$. Let $\bar{C}$ be the commutator ideal of $R=K_{+}\langle V\rangle / I$. The vector space $\bar{C}^{d} / \bar{C}^{d+1}$ is both left and right module over $\bar{R}=R / \bar{C} \cong K_{+}[V]$. We claim that $\bar{C}^{d} / \bar{C}^{d+1}=D_{d}+E_{d}$, where $D_{d}$ is a finitely generated left $\bar{R}$-module and $E_{d}$ is a finitely generated right $\bar{R}$-module $(d=1,2, \ldots)$. In order to see this we consider the following finite set of polynomials in $\bar{C}^{d} / \bar{C}^{d+1}$ :

$$
y_{1}^{(0)} \ldots y_{n}^{(0)}\left[z_{1}^{(1)}, z_{2}^{(1)}\right] y_{1}^{(1)} \ldots y_{s_{1}}^{(1)}\left[z_{1}^{(2)}, z_{2}^{(2)}\right] y_{1}^{(2)} \ldots y_{s_{d-1}}^{(d-1)}\left[z_{1}^{(d)}, z_{2}^{(d)}\right] y_{1}^{(d)} \ldots y_{p}^{(d)},
$$

where $n \leq N-1, p \leq M-1$ ( $N, M$ are from Proposition 5.3), $s_{1}+\ldots+s_{d-1} \leq$ $N(d, m)\left(N(d, m)\right.$ is from Lemma 5.4) and $y_{i}^{(j)}, z_{k}^{(l)}$ are any variables from the set $\left\{x_{1}, \ldots, x_{m}\right\}$. Let $D_{d}$ (respectively $E_{d}$ ) be the left (respectively right) $R$-module generated by this set. Clearly $\bar{C}^{d} / \bar{C}^{d+1}$ is spanned by the polynomials

$$
y_{1}^{(0)} \ldots y_{q}^{(0)}\left[z_{1}^{(1)}, z_{2}^{(1)}\right] y_{1}^{(1)} \ldots y_{t_{1}}^{(1)}\left[z_{1}^{(2)}, z_{2}^{(2)}\right] y_{1}^{(2)} \ldots y_{t_{d-1}}^{(d-1)}\left[z_{1}^{(d)}, z_{2}^{(d)}\right] y_{1}^{(d)} \ldots y_{r}^{(d)},
$$

where there is no restriction on $q, r, t_{1}, \ldots, t_{d-1}$. If $t_{1}+\ldots+t_{d-1} \geq N(d, m)$, then by Lemma 5.4 this polynomial can be expressed as a linear combination of similar 
polynomials with less than $N(d, m)$ variables among the commutators. If for some of the new polynomials $q \geq N$ and $r \geq M$, then by Proposition 5.3 we may express it as a linear combination of similar polynomials with $r \leq M-1$ and it is contained in $D_{d}$. If $q \leq N-1$ (respectively $r \leq M-1$ ), then we see immediately that the above polynomial is contained in $E_{d}$ (respectively $D_{d}$ ). This completes the proof of the claim.

Our assumptions clearly imply that the action of $G$ on $\bar{R}$ satisfies conditions (i) and (ii), hence by Corollary 2.3 the left $\bar{R}^{G}$-module $D_{d}^{G}$ and the right $\bar{R}^{G}$-module $E_{d}^{G}$ are finitely generated. Since $I$ is not contained in $C^{2}$, by [17] the T-ideal $I$ contains a polynomial $[z, x]^{k}\left(y(\operatorname{ad}[z, x])^{l}\right)[z, x]^{n}$ for suitable $k, l, n$. The matrix unit substitution $x \rightarrow e_{12}, y \rightarrow e_{12}, z \rightarrow e_{21}$ shows that this polynomial is not contained in the T-ideal of the identities of the $2 \times 2$ matrix algebra. Additionally the above polynomial is proper (i.e. it is a linear combination of products of commutators), so the T-ideal it generates in $K_{+}\left\langle x_{1}, x_{2}, \ldots\right\rangle$ is a non-matrix T-ideal in the free unitary algebra $K\left\langle x_{1}, x_{2}, \ldots\right\rangle$. Hence we may conclude from the remarks at the beginning of Section 4 that $\bar{C}^{k}=0$ for sufficiently large $k$. Now we may take a finite set of elements in $R^{G}$ such that their images generate $\bar{R}^{G}$ as a $K$-algebra (by the commutative Hilbert-Nagata theorem), $D_{d}^{G}$ as left $\bar{R}^{G}$-module and $E_{d}^{G}$ as a right $\bar{R}^{G}$-module $(d=1,2, \ldots, k-1)$. Clearly these elements generate $R^{G}$ as a $K$-algebra.

The above theorem has the following immediate consequence, which is in the flavour of Corollary 3.2.

Corollary 5.6. Let $R$ be a finitely generated $K$-algebra such that $R$ satisfies a polynomial identity not contained in $U$ and a polynomial identity not contained in $C^{2}$. Let $G$ act on $R$ such that (i) holds, and assume that there exists a finite dimensional subspace $V$ of $R$ generating $R$ as an algebra such that the action of $G$ on $K[V]$ satisfies (ii). Then $R^{G}$ is finitely generated. (In particular, if $G$ is a reductive linear algebraic group acting rationally on $R$, then $R^{G}$ is finitely generated.)

Remark. Obviously, Theorem 4.1 holds also in the case when $I$ is a T-ideal of the free non-unitary algebra $K_{+}\langle V\rangle$, provided that $I$ contains some power of $C$.

By the above theorem we have examples of non-Noetherian algebras for which the Hilbert-Nagata theorem holds.

\section{ACKNOWLEDGEMENTS}

Most of this research was done when the first author used the Exchange Program between the Hungarian and Bulgarian Academies of Sciences and visited the Institute of Mathematics and Informatics of the Bulgarian Academy of Sciences. He is very thankful for the warm hospitality.

\section{REFERENCES}

1. G. Almkvist, W. Dicks, E. Formanek, Hilbert series of fixed free algebras and noncommutative classical invariant theory, J. Algebra 93 (1985), 189-214. MR 86k:16001

2. S.A. Amitsur, The T-ideals of the free ring, J. London Math. Soc. 30 (1955), 470-475. MR 17:122c

3. M.F. Atiyah, I.G. Macdonald, Introduction to Commutative Algebra, Addison-Wesley Publ. Comp., Reading, Mass., 1969. MR 39:4129

4. J.A. Dieudonné, J.B. Carrell, Invariant Theory, Old and New, Academic Press, New YorkLondon, 1971. MR 43:4828 
5. V. Drensky, On the Hilbert series of relatively free algebras, Comm. Alg. 12 (1984), 2335-2347. MR 86f: 16005

6. V. Drensky, Computational techniques for PI-algebras, in "Topics in Algebra", Part 1 "Rings and Representations of Algebras", Banach Center Publ. 26, Polish Scientific Publishers, Warshaw, 1990, 17-44. MR 93e:16036

7. V. Drensky, Commutative and noncommutative invariant theory, in "Mathematics and Education in Mathematics", Union of Bulgarian Mathematicians, Sofia, 1995, 14-50.

8. E. Formanek, Noncommutative invariant theory, Contemp. Math. 43 (1985), 87-119. MR 87d:16046

9. P.J. Higgins, Lie rings satisfying the Engel condition, Proc. Cambridge Phil. Soc. 50 (1954), 8-15. MR 15:596b

10. G. Higman, On a conjecture of Nagata, Proc. Cambridge Phil. Soc. 52 (1956), 1-4. MR $17: 453 \mathrm{c}$

11. V.K. Kharchenko, Noncommutative invariants of finite groups and Noetherian varieties, J. Pure Appl. Alg. 31 (1984), 83-90. MR 85j:16052

12. O.G. Kharlampovich, M.V. Sapir, Algorithmic problems in varieties, Intern. J. Algebra and Computation 5 (1995), 379-602. MR 96m:20045

13. A.N. Krasil'nikov, Finite basis property of some varieties of Lie algebras, (Russian), Vestnik. Moskov. Univ. Ser. I, No 2 (1982), 34-38. MR 83i:17019

14. V.N. Latyshev, A generalization of Hilbert's theorem on the finiteness of bases, (Russian), Sibirsk. Mat. Zhur. 7 (1966), 1422-1424.

15. I.V. L'vov, Maximality conditions in algebras with identity, (Russian), Algebra i Logika 8 (1969), 449-459. MR 43:4853

16. Yu.N. Mal'tsev, On varieties of algebras, (Russian), Algebra i Logika 15 (1976), 579-584. MR 58:5457

17. L.A. Vladimirova, Codimensions of T-ideals containing an identity of fourth degree, (Russian), Serdica 14 (1988), 82-94. MR 89m:16025

18. N. Vonessen, Actions of linearly reductive groups on affine PI-algebras, Mem. Amer. Math. Soc. No. 414 (1989). MR 90i:16014

19. E.I. Zelmanov, Engelian Lie algebras, (Russian), Sibirsk. Mat. Zhur. 29 (1988), 112-117. MR 90a: 17010

Mathematical Institute of the Hungarian Academy of Sciences, Budapest, P.O. Box 127, H-1364, Hungary

E-mail address: domokos@math-inst.hu

Institute of Mathematics and Informatics, Bulgarian ACAdemy of Sciences, Acad. G. Bonchev Str., Block 8, 1113 Sofia, Bulgaria

E-mail address: drensky@math.acad.bg 